\title{
Equações de predição da aptidão cardiorrespiratória sem testes de exercício e sua aplicabilidade em estudos epidemiológicos: revisão descritiva e análise dos estudos
}

\author{
Geraldo de Albuquerque Maranhão Neto ${ }^{1,3,4}$ e Paulo de Tarso Veras Farinatti ${ }^{2}$
}

\section{RESUMO}

Aptidão cardiorrespiratória reduzida é considerada fator de risco independente para o óbito por todas as causas, mas principalmente por doença coronariana. Devido a essa importância e à dificuldade de avaliá-la através de testes de exercícios, formas alternativas de avaliação foram sugeridas, envolvendo equações de predição sem a necessidade de realização de exercícios. O presente estudo objetivou descrever e analisar criticamente esses modelos e, principalmente, sua aplicabilidade em estudos epidemiológicos. Foi realizada revisão sistemática de artigos publicados entre 1966 e 2003. Ao todo, 24 estudos foram selecionados obedecendo aos critérios de inclusão. Apenas cinco estudos relataram o erro padrão da estimativa (EPE), a equação completa, apresentam maior número amostral e, principalmente, realizaram a validação cruzada; além disso, estão entre os que apresentam maior valor de $\mathrm{R}^{2}$ ajustado, o que ratifica a qualidade e a força de predição dos mesmos. Conclui-se que, em princípio, os modelos sem exercícios podem constituir alternativa viável para avalia-

1. Programa de Pós-Graduação em Saúde Coletiva, Departamento de Epidemiologia, Instituto de Medicina Social, Universidade do Estado do Rio de Janeiro.

2. Laboratório de Atividade Física e Promoção da Saúde, Instituto de Educação Física e Desportos, Universidade do Estado do Rio de Janeiro, Rio de Janeiro, RJ.

3. Escola de Educação Física, Centro Universitário da Cidade (UniverCidade), Rio de Janeiro, RJ.

4. Departamento de Educação Física, Escola de Reabilitação, Universidade Católica de Petrópolis (UCP), Petrópolis, RJ.

Recebido em 19/6/03

2 versão recebida em 21/9/03

Aceito em 27/9/03

Endereço para correspondência:

Laboratório de Atividade Física e Promoção da Saúde

Instituto de Educação Física e Desportos

Universidade do Estado do Rio de Janeiro

Rua São Francisco Xavier, 524, sala 8.133-F

20550-013 - Rio de Janeiro, RJ, Brasil

labsau@uerj.br ção da aptidão cardiorrespiratória em estudos epidemiológicos. No entanto, são poucas as equações disponíveis cuja validação permite grau aceitável de generalização.

Palavras-chave: Aptidão física. Análises de regressão. Epidemiologia.

\section{RESUMEN}

Ecuaciones de predicción de la aptitud cardiorespiratoria sin test de ejercicios y su aplicabilidad a los estudios epidemiológicos: revisión descriptiva y análisis de estudios

Una reducida aptitud cardiorrespiratoria es considerada como factor de riesgo independiente para el óbito por todas sus causas, pero principalmente por enfermedad coronaria. Debido a esa importancia y a la dificultad de evaluarla a través de tests de ejercicios, fueron sugeridas formas de evaluación alternativas, envolviendo ecuaciones de predicción sin la necesidad de realizar ejercicios físicos. El presente estudio se centró en describir y analizar de forma crítica esos modelos y, principalmente, en su aplicabilidad en estudios epidemiológicos. Se realizó una revisión sistemática de los artículos publicados entre 1996 y 2003. En total, fueron seleccionados 24 de ellos, obedeciendo a criterios de inclusión. Solamente cinco artículos reportaron el error padrón de estimación (EPE), la ecuación completa, presentando un número mayor de muestras y principalmente realizaron una verificación cruzada; además de eso, figuran entre los que presentan mayor valor de $R^{2}$ ajustado, lo que ratifica la calidad y la fuerza de predicción de los mismos. Concluyéndose en primer lugar, que los modelos sin ejercicios pueden constituirse en una alternativa viable para la evaluación de la aptitud cardiorrespiratoria en estudios epidemiológicos. A pesar de esto, las ecuaciones disponibles, cuya validez permita un grado aceptable de generalización, son pocas.

Palabras clave: Aptitud física. Análisis de regresión. Epidemiología. 


\section{INTRODUÇÃO}

A aptidão cardiorrespiratória é considerada o componente da aptidão física relacionado à saúde que descreve a capacidade dos sistemas cardiovascular e respiratório em fornecer oxigênio durante uma atividade física contínua ${ }^{1,2}$. O risco de morbimortalidade por doenças crônico-degenerativas, entre elas, doença arterial coronariana, hipertensão arterial sistêmica, diabetes mellitus e alguns tipos de câncer, tem sido associado a baixos níveis de aptidão cardiorrespiratória e atividade física ${ }^{3-9}$. Seria, então, importante avaliar a capacidade cardiorrespiratória em nível populacional.

A utilização da aptidão cardiorrespiratória como variável de exposição em estudos epidemiológicos, porém, é limitada pelos elevados custos, por dificuldades técnicas de operação e pelo tempo gasto em sua mensuração ${ }^{10,11}$. Essas circunstâncias têm motivado o desenvolvimento de métodos mais simples, substituindo os testes de exercício tanto máximo quanto submáximo por equações de regressão linear múltipla para predizer a aptidão cardiorrespiratória a partir de características físicas e hábitos de vida ${ }^{11-14}$. Dispor de técnicas desse tipo, mais simples, menos onerosas e de rápida aplicação, favoreceria a utilização da aptidão cardiorrespiratória como variável de exposição em estudos epidemiológicos, principalmente em localidades com pouca infra-estrutura ${ }^{12,15}$. Isso posto, o objetivo do presente estudo foi fazer um levantamento dos estudos envolvendo equações de predição da aptidão cardiorrespiratória sem exercício, descrever a evolução desse tipo de técnica e analisar as características das equações desenvolvidas, principalmente no que toca à sua qualidade.

\section{METODOLOGIA}

Os artigos potencialmente úteis foram obtidos de referências bibliográficas de artigos e livros publicados (modo manual) e através de pesquisa bibliográficas nos bancos de dados Medlars online (Medline) Silver Platter e Literatura Latino-americana e do Caribe de Informação em Ciências da Saúde (Lilacs). O estudo de revisão foi restrito ao período de janeiro de 1966 até dezembro de 2002 no Medline e, no Lilacs, de 1982 até 2002.

Todos os artigos potencialmente úteis obtidos através da busca eletrônica tiveram seus resumos extraídos e foram analisados de maneira independente por dois revisores, um deles da área de atividade física aplicada à saúde com conhecimento sobre o tema em estudo e o outro, um epidemiologista com conhecimento sobre revisão sistemática. O principal critério de inclusão utilizado foi o de coletar estudos que tinham como objetivo a predição da aptidão cardiorrespiratória sem testes de exercício, baseados em variáveis de simples aferição, úteis para estudos populacionais, tais como: peso, estatura, medidas antropométricas e nível de atividade física. A partir da obtenção e leitura dos artigos, suas referências bibliográficas foram rastreadas à procura de outros artigos potencialmente utilizáveis. Esse trabalho repetiu-se tantas vezes quanto necessário, até haver a convicção de que nenhuma das referências obtidas continha estudos que já não tivessem sido identificados.

\section{EVOLUÇÃO HISTÓRICA DAS EQUAÇÕ̃ES SEM EXERCÍCIO}

A fase de revisão permitiu localizar 24 artigos que atenderam aos critérios de inclusão, todos estudos originais publicados desde 1967. A evolução da definição dos objetivos e das características metodológicas dos estudos tornou evidente o esforço para a produção de equações mais acuradas da capacidade cardiorrespiratória. Em vista disso, os estudos serão apresentados em ordem cronológica. Com o objetivo de enriquecer a discussão sobre o tema, durante o processo de descrição dos estudos achou-se por bem apresentar outras pesquisas que tenham, em determinada época, auxiliado no conhecimento sobre o tema ou verificado a validação cruzada das equações de predição originais.

As primeiras pesquisas que sugerem a avaliação do consumo máximo de oxigênio, através de variáveis coletadas sem a necessidade de testes de exercício, datam do final da década de 60. Na ocasião, as pesquisas caracterizavam-se pela mensuração da quantidade de potássio corporal, por técnica radiodiagnóstica de tecido muscular ${ }^{16,17}$. Na utilização dessa técnica admite-se que a concentração de potássio na massa isenta de gordura seja constante. Com isso, ao ser estabelecida a quantidade de potássio corporal, torna-se possível desenvolver predições quanto à massa isenta de gordura. A justificativa para a verificação da massa corporal magra residia no fato de que um indivíduo fisicamente ativo tenderia a apresentar correlação positiva entre aptidão cardiorrespiratória e massa muscular.

Shephard et al..$^{18}$ publicaram, em 1971, o primeiro estudo com o objetivo de predizer a aptidão cardiorrespiratória através de regressão múltipla e sem a realização de testes de exercício. Trinta e sete medidas antropométricas e índices de força corporal foram coletados em 46 crianças e adolescentes de ambos os sexos, parte de uma amostra randomizada de escolares de Toronto (Canadá). As equações mais promissoras e de maior aplicabilidade para outros estudos foram aquelas baseadas na área de superfície corporal (calculada através das medidas de peso e estatura), além da dobra cutânea de coxa e idade. Os autores chegaram à conclusão de que, em crianças, a aptidão cardiorres- 
piratória poderia ser convenientemente predita pelo método proposto. Dois anos depois, Bruce et al. ${ }^{19}$ estabeleceram algumas equações de predição da aptidão cardiorrespiratória com e sem testes de exercício, demonstrando que esta também poderia ser predita através de variáveis como sexo, idade, peso e o hábito de fazer atividade física, através da aplicação da análise de regressão múltipla passo a passo (stepwise). Esse estudo foi o primeiro a utilizar adultos e a demonstrar que a aptidão cardiorrespiratória poderia ser predita não apenas através de dados antropométricos, mas também a partir de variáveis comportamentais, como a atividade física cotidiana.

Entre os estudos utilizando variáveis antropométricas, seguindo a linha de Shephard et al..$^{18}$, destacam-se os de Mayhew e Gifford ${ }^{20}$ e Bonen et al..$^{21}$, em 1975 e 1979, respectivamente. No primeiro $^{20}$ foram estudados 31 meninos entre sete e nove anos de idade, estimando-se o $\dot{\mathrm{V}}_{2}$ máximo através de várias medidas antropométricas. Inicialmente, a correlação simples do $\dot{\mathrm{V}}_{2}$ máximo com as medidas era realizada. Após esse procedimento, a análise de regressão múltipla passo a passo foi utilizada para selecionar as equações mais representativas. Mais uma vez, as medidas mais significativas eram relacionadas aos membros inferiores: volume e dobras cutâneas de perna e coxa apresentaram o maior coeficiente de explicação $\left(\mathrm{R}^{2}=0,64\right)$. No segundo ${ }^{21}$, também com crianças e adolescentes, os autores verificaram em 100 meninos de sete a 15 anos a força de predição das variáveis idade, peso e estatura. De acordo com os autores, o alto coeficiente apresentado $\left(R^{2}=0,88\right)$ e o fato de as crianças não apresentarem boa adaptação à maioria dos testes de exercícios reforçariam ainda mais a proposição de que equações de predição com medidas de simples coleta seriam uma ótima alternativa ao cálculo indireto da potência aeróbia. Enfim, em 1978, Taylor et al..$^{22}$ tentaram predizer o tempo total gasto em minutos durante teste ergométrico em esteira, através do total de escores obtidos com o Minnesota Leisure Time Physical Activity (MLTPA), desenvolvido pela Universidade de Minnesota. O MLTPA busca levantar as atividades físicas praticadas no período correspondente ao último ano. A equação não apresentou associação muito forte $\left(R^{2}=0,27\right)$, o que poderia ser um indício de que apenas o histórico de atividade física não deveria ser utilizado para a predição da aptidão cardiorrespiratória.

A partir da década de 80 , apenas três estudos realizados na Índia buscaram predizer a aptidão cardiorrespiratória utilizando somente variáveis antropométricas. No primeiro deles ${ }^{23}$ foram realizadas 27 medidas antropométricas, utilizando-se a regressão múltipla passo a passo para verificar quais variáveis poderiam predizer significativamente o $\dot{\mathrm{vO}}_{2}$ máximo de 120 mulheres e homens. Quatro variáveis permaneceram no modelo final: peso, estatura, diâmetro de cotovelo e dobra cutânea de peitoral. Em outro estudo, com 70 indivíduos do sexo masculino tendo entre 11 e 18 anos de idade, Verma et al. ${ }^{24}$ verificaram uma relação do $\dot{\mathrm{V}}_{2}$ máximo com idade, peso e estatura, identificando maior poder de explicação $\left(R^{2}=0,81\right)$ em equação de regressão com base exclusiva no peso corporal. Finalmente, com um grupo de 146 homens, Verma et al. ${ }^{25}$, já em 1998, verificaram como a aptidão cardiorrespiratória poderia ser predita a partir da idade, estatura e peso corporal. Uma equação envolvendo idade e peso corporal foi então obtida. Os dois estudos indianos realizados com adultos apresentaram coeficientes explicativos semelhantes em suas equações (respectivamente, 0,29 e 0,35 ), sugerindo que a utilização de variáveis antropométricas talvez não se aplicasse tão bem em adultos quanto em crianças e adolescentes. Uma possibilidade a ser considerada, que justificaria o relativo sucesso da utilização exclusiva de variáveis antropométricas nas equações para crianças e adolescentes, é o fato de a idade biológica na infância associar-se diretamente com as proporções corporais. Não temos dados, porém, capazes de ratificar essa hipótese.

Em 1981, Leon et al. ${ }^{26}$ predisseram o tempo gasto em teste máximo de esteira em 175 homens de meia-idade, valendo-se tanto de variáveis antropométricas quanto comportamentais. Esse trabalho foi o primeiro a propor a utilidade da predição da aptidão cardiorrespiratória sem testes de exercício para estudos epidemiológicos, baseando-se no aumento do número de evidências sobre baixa aptidão cardiorrespiratória e o risco de morte por doença arterial coronariana. Foram selecionadas 11 variáveis preditivas: idade, índice de atividades intensas baseando-se no MLTPA, índice de massa corporal (IMC), tabagismo passado ou atual, desempenho habitual em atividade ocupacional que fizesse suar ou provocasse dispnéia, número de copos de café, chá ou refrigerante de cola por semana, hábito de fumar cachimbo ou charuto, atividades de lazer que provocassem suor ou dispnéia, duração média do sono em horas e frequiência cardíaca de repouso. Os autores concluíram que boa predição da capacidade cardiorrespiratória poderia ser obtida através de questionários padronizados, em conjunto com medidas físicas simples, apesar de o valor encontrado para o coeficiente de determinação ter sido moderado $\left(R^{2}=0,53\right)$.

Utilizando a atividade física auto-referida para predizer o consumo máximo de oxigênio, Siconolfi et al. ${ }^{27}$ observaram, em 36 homens e 32 mulheres, que a força de predição das equações era maior ao aferirem a intensidade da atividade física praticada, ao invés de simplesmente checarem se os indivíduos a praticavam ou não. A partir desse estudo, a intensidade da atividade física praticada passaria a 
ser uma variável constante e de grande importância nos estudos de predição. Por exemplo, dois anos após, Milesis $^{28}$ estimou o tempo de permanência em teste máximo de esteira em 126 homens e 70 mulheres, com base nas variáveis sexo, idade, índice recíproco ponderal (estatura dividida pela raiz cúbica do peso), nível de atividade física categorizado de 1 a 5 (sedentário, pouco ativo, ativo, muito ativo e atleta), histórico de fumo categorizado de 0 a 2 (nunca fumou, fumante de menos de 20 cigarros/dia e fumante de mais de 20 cigarros/dia) e frequiência cardíaca de repouso. Kohl et al. ${ }^{15}$, através de questionário enviado pelo correio, predisseram o tempo máximo (em minutos) de desempenho em um teste de esteira aplicado a 375 pessoas com média de idade de 47,1 anos. Na equação preditiva constavam a idade e variáveis relativas à intensidade da atividade física, tais como um escore de participação em atividades como caminhar e correr e a freqüência com que realizavam atividades com intensidade suficiente para provocar a transpiração.

Um ano depois, em estudo importante devido ao amplo universo amostral, Blair et al..$^{12}$ desenvolveram uma equação para a predição do tempo em teste de esforço máximo em esteira de 15.627 homens $(42,5 \pm 9,5$ anos) e 3.943 mulheres $(42,1 \pm 10,7$ anos). Dividindo os indivíduos em cinco grupos por faixa de idade, desde 20 a 29 anos até mais de 60 , obtiveram equações preditivas com coeficientes de explicação que variavam de 0,49 a 0,60 em homens e de 0,20 a 0,49 em mulheres. As equações constavam das seguintes variáveis: IMC, freqüência cardíaca de repouso, um índice de atividade física de lazer no último mês (sendo que 1 equivaleria a nenhuma atividade física praticada no último mês e 5 a andar, correr ou trotar mais de $32 \mathrm{~km}$ por semana) e tabagismo (se o indivíduo fumava ou não). Nesse estudo consta uma evidência adicional, a análise longitudinal dos dados, destacando que alterações no IMC e na frequiência de repouso contribuíram entre 14 e 19\% na mudança do tempo em esteira.

Estudos para predizer a aptidão cardiorrespiratória em indivíduos coronariopatas tiveram como pioneiros Lee et $a l .^{29}$, em fins dos anos 80, através da assim chamada Escala de Atividades Específicas (Specific Activity Scale SAS $)^{30}$. Lee et al. ${ }^{29}$ demonstraram, em 36 pacientes coronariopatas e indivíduos saudáveis, que a informação autoreportada da capacidade de realizar atividades diárias (como vestir-se, tomar banho ou subir um lance de escadas) poderia ter um papel de complemento ao teste de esforço na predição da capacidade cardiorrespiratória. Inclusive, isso possibilitaria à equipe de saúde até mesmo decidir se o indivíduo deveria ou não fazer o teste, de acordo com as limitações relatadas. Pouco depois, visando à utilização em estudos epidemiológicos longitudinais, Hlatky et al. ${ }^{31}$ va- lidaram uma equação preditiva da aptidão cardiorrespiratória sem o emprego de testes de exercício em pacientes coronariopatas. Inicialmente, o consumo máximo de oxigênio foi correlacionado com a capacidade funcional de 50 pacientes, utilizando-se o Índice do Nível de Atividade do Centro Médico da Universidade de Duke (Duke Activity Status Index - DASI) como critério. Esse índice era composto de 12 itens, englobando atividades relacionadas ao cuidado pessoal, atividades no lar, atividades sexual e recreativa, ponderadas por seu gasto metabólico individual medido em METs. A correlação de Spearman foi alta $(0,80)$. No entanto, como esse primeiro grupo foi entrevistado, mais 50 outros indivíduos passaram pelo mesmo questionário, só que agora autopreenchido, tendo sido verificada correlação mais baixa $(0,58)$. Ao final desse processo, uma equação de regressão simples foi gerada com os dados do primeiro e segundo grupos. De acordo com os autores, mais estudos precisariam ser realizados para verificar se o DASI é sensível para detectar mudanças longitudinais. Além disso, eles não acreditam que o questionário possa substituir o teste de esforço, mesmo consistindo em boa ferramenta para detectar a autonomia de pacientes com doença arterial coronariana.

Em 1990, Jackson et al. ${ }^{13}$ desenvolveram dois modelos de predição da aptidão cardiorrespiratória com as variáveis sexo, idade, composição corporal e atividade física auto-referida (de 0 a 7 , de acordo com a intensidade, sendo 0 aquela pessoa que não participa de nenhuma atividade física ou esporte no último mês e 7 aquele que corre mais de 10 milhas ou gasta mais de três horas por semana em atividade física semelhante à corrida). Um dos modelos utilizava o IMC como indicador de composição corporal e o outro, o percentual de gordura $(\% \mathrm{G})$ predito pela medida de dobras cutâneas. Tanto o modelo com o \%G $\left(\mathrm{R}^{2}=0,66\right)$ quanto o com o IMC $\left(\mathrm{R}^{2}=0,62\right)$ demonstraram, segundo os autores, boa predição em 1.393 homens e 150 mulheres com idades entre 20 e 70 anos. A acurácia da equação foi confirmada quando a mesma foi aplicada na amostra de validação cruzada, contando com 423 homens e 43 mulheres saudáveis e hipertensos. Os coeficientes de correlação de Pearson entre os valores preditos e observados no modelo com $\%$ G e IMC foram de 0,82 e 0,79 , respectivamente. Apenas em sujeitos muito bem condicionados (com $\mathrm{V}_{2}$ máximo $\geq 55 \mathrm{ml} \cdot \mathrm{kg}^{-1} \cdot \mathrm{min}^{-1}$ ) os modelos demonstraram tendência a subestimar a aptidão. Porém, pessoas com essa característica encontram-se bem acima da média populacional, não afetando a aplicabilidade dos modelos para estudos com grande número amostral. Esses modelos mostraram-se mais acurados do que o modelo de predição em esteira de Åstrand e Ryhming ${ }^{32}$, que utilizaram a freqüência cardíaca medida durante o exercício submáximo em 
esteira. Tais resultados confirmaram as idéias já defendidas por Shephard et al. ${ }^{18}$, de que modelos sem exercício poderiam ser mais acurados do que testes físicos submáximos. Além disso, esse foi o primeiro estudo em que os pesquisadores demonstraram preocupação especial com procedimentos de validação cruzada.

O interesse pelos modelos propostos por Jackson et al. ${ }^{13}$ rendeu dois estudos, com o objetivo de verificar a acurácia das equações propostas em amostras com características distintas: Kolhorst e Dolgener ${ }^{33}$ verificaram a validade dos modelos em 69 estudantes universitários fisicamente ativos. O estudo contou com 28 homens e 41 mulheres, tendo, em média, $21 \pm 2$ anos de idade. Ao aplicarem a correlação de Pearson para comparar os resultados da aptidão cardiorrespiratória medida e predita, os autores observaram que os dois modelos de Jackson et al. ${ }^{13}$ não apresentaram boa correlação $(r=0,72)$, ratificando a conclusão do estudo original de terem aplicabilidade limitada em pessoas com alta aptidão. Em 1996, Williford et al. ${ }^{34}$ verificaram a validação cruzada dos modelos sem exercício propostos ${ }^{13} \mathrm{em}$ uma amostra de 165 mulheres, uma vez que a amostra da validação cruzada do estudo original era pequena $(n=43)$. Tanto a equação com o IMC quanto com o \% mostraram boa correlação ( $\mathrm{r}=0,81$ e 0,86 , respectivamente), ratificando a acurácia dessas equações também em mulheres entre 18 e 45 anos. $\mathrm{O}$ modelo conseguiu predizer a aptidão de $87 \%$ das mulheres com $\dot{V}_{2}$ máximo $<32 \mathrm{ml} \cdot \mathrm{kg}^{-1} \cdot \mathrm{min}^{-1}$, valor que apresentaria maior associação com risco de mortalidade por todas as causas ${ }^{12}$, sugerindo a sua utilização em investigações de cunho epidemiológico.

Em 1992, Ainsworth et al..$^{10}$ desenvolveram uma equação de predição da aptidão cardiorrespiratória através de uma pergunta sobre a frequiência com que o indivíduo realizava atividades físicas intensas por mais de 15 minutos, além de outras variáveis de fácil aferição, como idade, sexo e IMC. Para tanto, contaram com um número amostral não muito grande, de 27 homens e 47 mulheres entre 21 e 59 anos de idade. Uma interessante característica desse estudo é que, para os autores chegarem à questão mais adequada sobre atividade física, aplicaram vários questionários de atividade física utilizados em pesquisa epidemiológi$\mathrm{ca}^{35-39}$. Ao final, apenas uma pergunta sobre a participação regular em atividades físicas mais intensas ${ }^{37}$ permaneceu no modelo, reforçando a idéia de que a variável atividade física poderia ser aferida de forma simples para informar sobre a aptidão cardiorrespiratória, desde que a intensidade da atividade fosse levada em consideração, juntamente com outras variáveis. Dois anos depois, Myers et al. ${ }^{40} \mathrm{de}-$ senvolveriam uma equação para predizer o desempenho em teste máximo de esteira em 207 homens e cinco mulheres $(62 \pm 8$ anos $)$ coronariopatas, através dos resultados obtidos no questionário de Atividade Física Específica em Veteranos (Veteran Specific Activity Questionnaire - VSAQ) e da idade. Os participantes do estudo preencheram, no questionário, qual atividade física eram capazes de realizar sem sintomas limitadores de esforço (fadiga, desconforto no peito, dispnéia). Através da equação de regressão múltipla gerada, os autores desenvolveram um nomograma em que, a partir do número de METs definido pelo questionário e da idade do indivíduo, era predito o seu desempenho em esteira. De acordo com os autores, a equação não teria o objetivo de substituir o teste ergométrico, mas de possibilitar que a equipe de saúde tivesse noção da condição física do indivíduo, podendo adequar o teste a essa condição.

Whaley et al. ${ }^{41}$ desenvolveram outro modelo de predição da aptidão com as variáveis sexo, idade, frequiência cardíaca de repouso, peso, percentual de gordura, fumo (de 1 a 8 , de acordo com a freqüência e o número de cigarros, 1 para o não fumante e 8 para o que fuma mais de dois maços por dia) e atividade física auto-relatados (de 1 a 6 de acordo com a intensidade, sendo 1 aquele indivíduo com estilo de vida sedentário e 6 aquele altamente treinado que corre, pedala ou nada mais de 20 milhas por semana). Participaram do estudo 702 homens e 473 mulheres, e a equação preditiva demonstrou boa acurácia $\left(\mathrm{R}^{2}=0,72\right)$. Assim como o estudo de Jackson et al. ${ }^{13}$, esse também realizou a validação cruzada da equação. A correlação de Pearson entre valores preditos e medidos $(r=0,85)$ fez os autores considerarem a equação como válida. Ainda no mesmo ano, Heil et al. ${ }^{14}$ validaram um modelo de predição sem exercício com as variáveis sexo, idade e idade ${ }^{2}$, percentual de gordura e o escore do nível de atividade física utilizado por Jackson et al..$^{13}$, junto a 229 mulheres e 210 homens com 20 a 79 anos de idade $\left(R^{2}=0,77\right)$. A validação cruzada foi realizada em 65 indivíduos de características semelhantes às do grupo para o qual a equação foi validada. Segundo os autores, o resultado da correlação de Pearson foi bom $(r=0,85)$; contudo, a pequena amostra limita um pouco os resultados da validação cruzada. Apesar disso, a equação gerada reforça a idéia de que se pode, realmente, predizer a aptidão cardiorrespiratória a partir de algumas variáveis sugeridas por Jackson et al. ${ }^{13}$.

Em 1996, outro questionário de predição foi validado, baseando-se na limitação funcional de pacientes coronariopatas: o Questionário de Atividades Específicas (Specific Activity Questionnaire - SAQ), com 13 itens relacionados às atividades cotidianas ${ }^{42}$. Noventa e sete pacientes (sendo 12 mulheres) tiveram sua capacidade predita através do escore do $S A Q$, estatura, idade e peso $\left(R^{2}=0,50\right)$. A correlação de Pearson foi calculada entre o SAQ e outros questionários em relação à aptidão cardiorrespiratória, ob- 
tendo-se os seguintes resultados: $\mathrm{SAQ}(\mathrm{r}=0,71), \mathrm{SAS}^{29}(\mathrm{r}=$ $0,35), \operatorname{DASI}^{31}(r=0,62)$ e VSAQ $^{40}(r=0,66)$. Para os autores, isso demonstraria o potencial de utilização do SAQ em estudos com coronariopatas, caso o teste de esforço se revelasse dispendioso ou inviável. No mesmo ano, Cardinal ${ }^{43}$ publicou estudo em que verificou se as equações propostas por Jackson et al. ${ }^{13} \mathrm{e}$ Ainsworth et al. ${ }^{10}$ tinham associação entre si e com outros índices de atividade física, em 123 mulheres saudáveis (idade $=38,8 \pm 8,4$ anos). A conclusão foi de que tanto as equações $(r=0,80)$ quanto os índices de atividade física $(0,26$ a 0,74) tinham, em geral, boa correlação entre si e seguiam critérios semelhantes de classificação. Um ano depois, George et al. ${ }^{44}$ estabeleceram um modelo de predição adaptado para jovens estudantes fisicamente ativos, em amostra de 50 homens e mulheres com 18 a 29 anos de idade. Para aumentar a acurácia do modelo final $\left(R^{2}=0,72\right)$, já que as tentativas para predizer a aptidão de pessoas muito bem condicionadas haviam falhado até o momento, novas variáveis passaram a ser acrescentadas. Entre elas havia uma questão sobre a aptidão percebida em atividades como andar e correr, na qual as pessoas deveriam dizer em qual ritmo conseguiriam locomover-se sem ficar extremamente cansadas. Outra questão remetia ao histórico de atividade física, classificada de 0 a 10 dentro de um período de seis meses, ao invés do 0 a 7 em um histórico de um mês, conforme proposto no estudo de Jackson et $a l .{ }^{13}$. Os autores consideram ser esse o primeiro modelo sem necessidade de qualquer tipo de aferição, uma vez que peso e estatura para o cálculo do IMC foram auto-referidos. Nesse estudo, o processo de validação cruzada foi diferente dos anteriores. Ao invés de utilizar uma amostra do grupo inteiro em estudo, o que segundo os autores acabaria limitando a amostra, o método de soma dos quadrados dos resíduos preditos (PRESS) foi utilizado. Esse método permite a utilização de todos os indivíduos em estudo, tanto na validação quanto na validação cruzada. Para tanto, baseia-se no cálculo dos resíduos preditos para cada sujeito, enquanto o mesmo é excluído do modelo original ${ }^{45}$. A partir da soma dos quadrados desses resíduos, é possível calcular o $\mathrm{R}^{2}(0,71)$ e o erro padrão da estimativa, demonstrando-se a boa acurácia do modelo.

Em 1999, Mathews et al. ${ }^{11}$ propuseram um modelo e examinaram a sua sensibilidade para categorizar a aptidão cardiorrespiratória. Nesse sentido, os autores consideraram que o fato de isso não ter sido feito tenderia a limitar a aplicação de suas equações em estudos epidemiológicos. A categorização possibilitaria que estimativas de risco de doenças pudessem ser comparadas entre níveis de aptidão diferentes. Seguindo o exemplo de George et al. ${ }^{44}$, fizeram parte do modelo apenas variáveis auto-referidas, quais sejam: idade, idade ${ }^{2}$, sexo, atividade física reportada (con- forme proposto por Jackson et al. ${ }^{13}$ ), estatura e peso corporal $\left(\mathrm{R}^{2}=0,74\right)$. A acurácia de classificação por parte do modelo foi examinada na tabulação dos dados em categorias de idade e sexo, distribuídos em quintis de aptidão cardiorrespiratória medida e predita. A acurácia total de classificação do modelo foi modesta (36\%). Porém, $83 \%$ de todos os sujeitos foram classificados corretamente ou no mais próximo quintil. $\mathrm{O}$ erro extremo na classificação do menor para o maior quintil foi raramente observado $(0,13 \%)$, o que levou à conclusão de que os valores de aptidão preditos poderiam ser utilizados como variável de exposição em estudos epidemiológicos, quando o teste de esforço não fosse uma opção viável. Para o processo de validação cruzada, o método PRESS também foi utilizado, ratificando a validade do modelo $\left(\mathrm{R}^{2}=0,74\right)$.

Em estudo que parece ser, até a elaboração deste texto, o último publicado sobre a predição da aptidão cardiorrespiratória sem testes de exercício, Wu e Wang ${ }^{46}$ estabeleceram uma equação a partir da observação de 24 trabalhadores de ambos os sexos residentes em Taiwan. As variáveis que foram identificadas como significativas no modelo de regressão foram sexo, idade e IMC $\left(\mathrm{R}^{2}=0,77\right)$, ratificadas pelo processo de validação cruzada em uma pequena amostra $(\mathrm{N}=6)$. Os autores acreditaram que o modelo poderia ser adequado para uma população ocupacionalmente ativa. Todavia, a generalização dos resultados reportados esbarra na óbvia limitação representada pelo quantitativo amostral, por demais reduzido.

\section{ANÁLISE CRÍTICA DOS MODELOS REVISADOS}

Nos quadros 1 e 2, são apresentados os estudos, seu país de origem, amostra, gênero, faixa etária e as equações de predição, acompanhados do $\mathrm{R}^{2}$ ajustado e do erro padrão da estimativa (EPE). No quadro 1, encontram-se todos os estudos em que foi utilizado o $\mathrm{V}_{2}$ máximo como variável dependente, tanto relativo $\left(\mathrm{ml}^{\mathrm{kg}} \mathrm{kg}^{-1} \cdot \mathrm{min}^{-1}\right)$ quanto absoluto (1. $\left.\mathrm{min}^{-1}\right)$. Já no quadro 2, a variável dependente foi o tempo gasto durante um teste máximo de esteira (expresso em minutos) ou a intensidade máxima do teste (expressa em METs).

A regressão linear múltipla tem sido a análise estatística mais utilizada para a predição da aptidão cardiorrespiratória sem teste de exercícios. A sua utilização justifica-se quando o pesquisador pretende explicar quais variáveis contribuem para a predição da variável dependente (aptidão cardiorrespiratória) e a magnitude desta contribuição ${ }^{47}$. Em alguns estudos, apenas a regressão linear simples foi utilizada $^{24,27,29,31}$. Todos os artigos apresentaram o R da equação (coeficiente de correlação múltipla) ou o $\mathrm{R}^{2}$ (coeficiente de explicação), referentes à capacidade explicativa do modelo. A apresentação desses dados está de acordo com as 


\section{QUADRO 1}

\section{Equações de predição do $\mathrm{VO}_{2 \text { máximo }}$ sem a realização de exercícios}

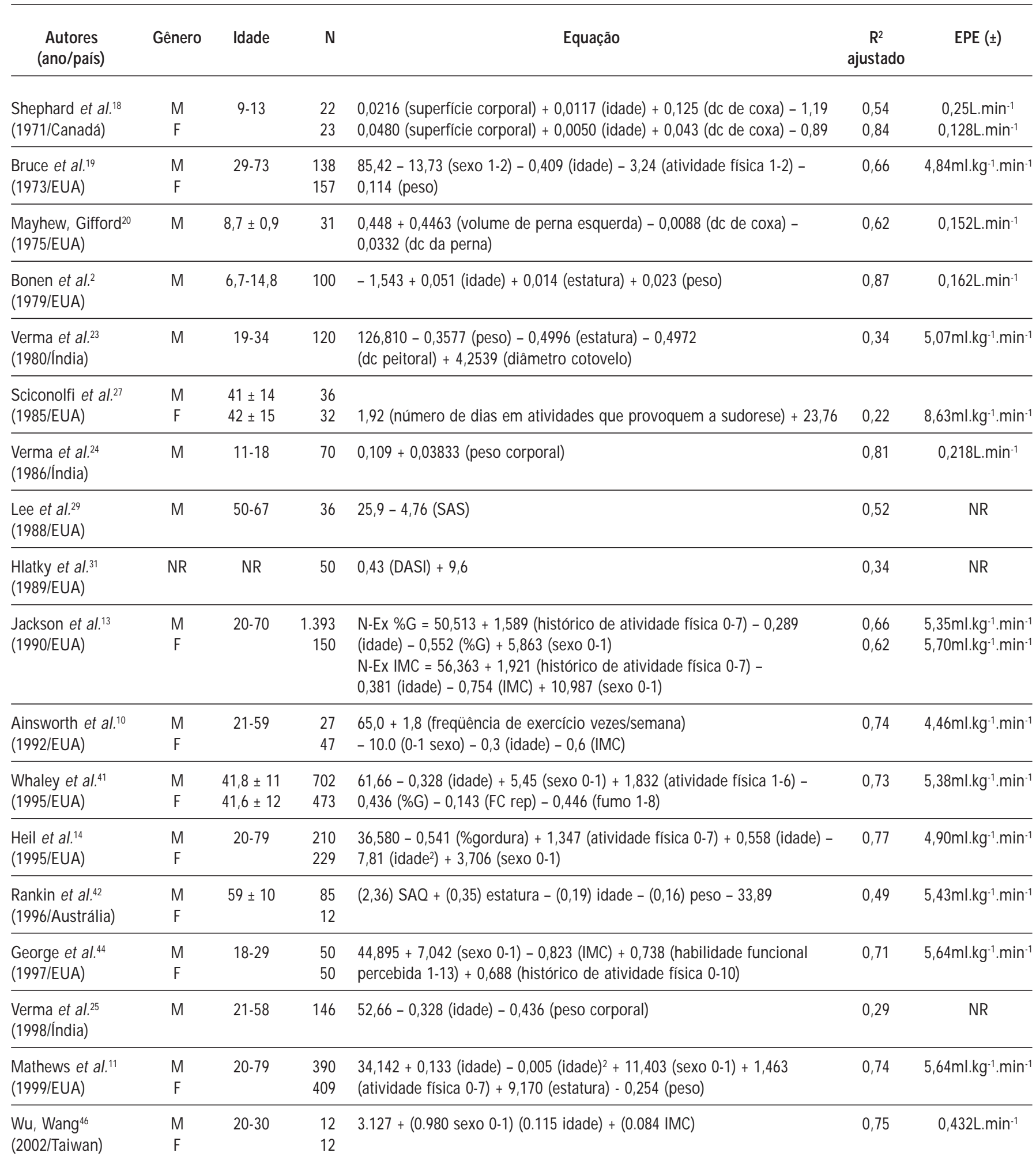

EPE - Erro padrão da estimativa; \#- Calculado a partir do R; M - masculino; F- feminino; dc - dobra cutânea; Sexo 1 - feminino; 2 - masculino ou 0 - feminino, 1 - masculino; SAS - Specific Activity Status; DASI - Duke Activity Status Index; SAQ - Specific Activity Questionnaire; IMC - índice de massa corporal; \%G - percentual de gordura. 
QUADRO 2

Equações de predição da duração ou intensidade máxima do teste em esteira sem a realização de exercícios

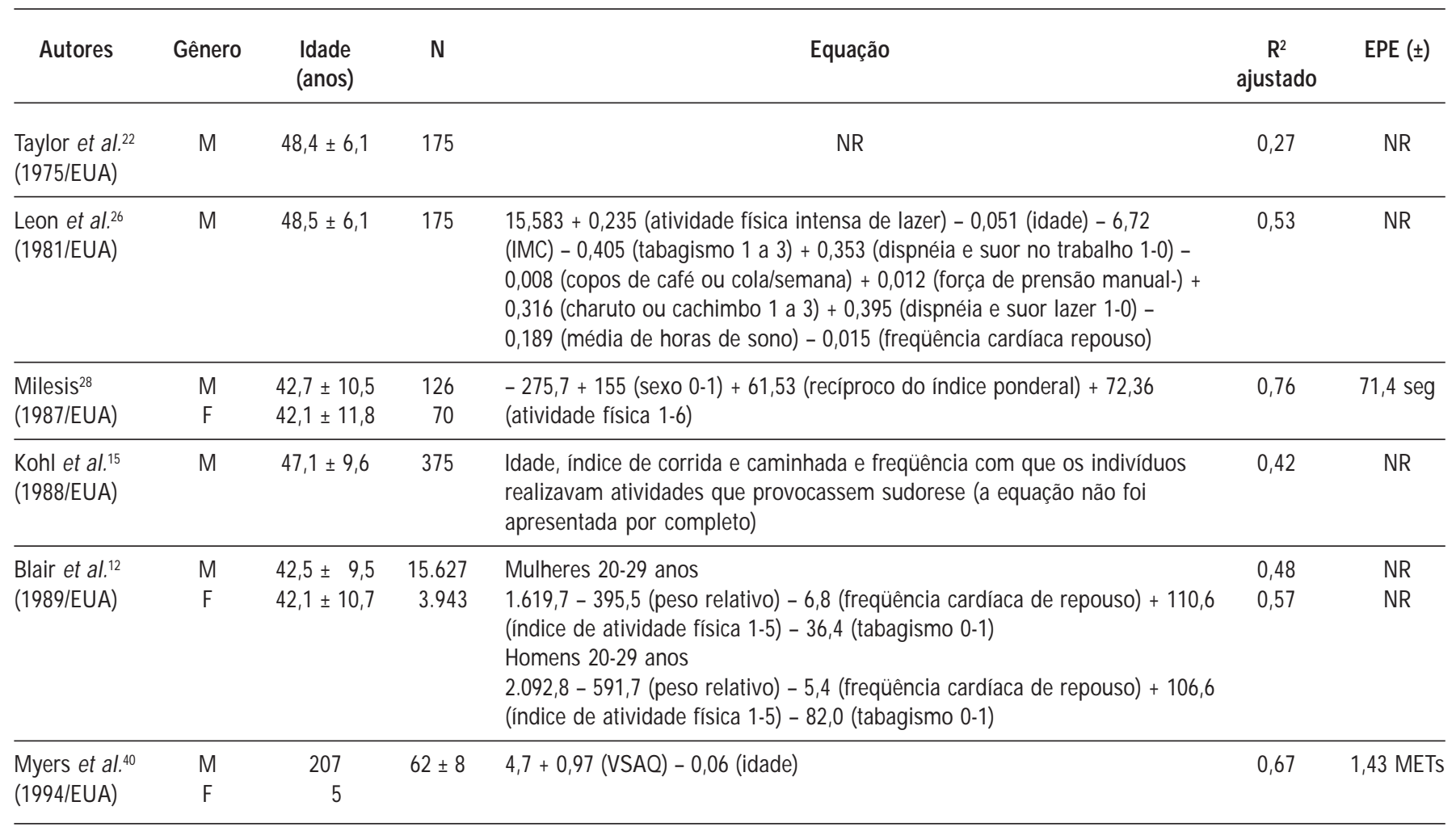

VSAQ - Veterans Specific Activity Questionnaire

recomendações encontradas na literatura ${ }^{48}$. $\mathrm{O} \mathrm{R}^{2}$ ajustado pode ser facilmente calculado e é útil para melhor análise dos modelos, já que não é influenciado pelo número de variáveis independentes. Por outro lado, o $\mathrm{R}^{2}$ tende a inflar em função da quantidade de variáveis introduzidas na equação. Calcula-se o $\mathrm{R}^{2}$ ajustado através da fórmula ${ }^{48}$

$$
R^{2} \text { ajustado }=1-\left[\left(1-R^{2}\right) n-1 / n-p\right],
$$

onde $n$ é o número amostral e $p$ o número de parâmetros. O cálculo do $\mathrm{R}^{2}$ ajustado justifica-se para que seja possível analisar e comparar a qualidade do ajuste de modelos preditivos com quantidades diferentes de variáveis. Com base nesse cálculo, pode-se afirmar que as equações que apresentarem maior valor do $\mathrm{R}^{2}$ ajustado são, igualmente, as com maior capacidade explicativa na amostra para a qual foram validadas. Quanto ao EPE, indica a variação não explicada pela linha de regressão, consistindo em uma medida da discrepância entre as variáveis observadas e preditas. Alguns autores consideram que a não indicação do EPE reduz a qualidade do estudo, principalmente quando não apresentam a equação completa ${ }^{48,49}$. O fato de alguns estudos não terem predito o consumo máximo de oxigênio (qua- dro 2) implica que as equações referem-se a uma variável independente duplamente indireta, o que afetaria ainda mais a qualidade das equações: afinal, o tempo gasto em teste máximo já é um indicador indireto da aptidão cardiorrespiratória. A eficiência mecânica também é um fator que interfere no resultado, independentemente do $\dot{\mathrm{V}}_{2}$. Além disso, outros estudos ${ }^{21,23-25}$ relataram que o consumo máximo de oxigênio foi predito e não medido, o que afetaria ainda mais a qualidade dessas predições.

Os cinco estudos que têm maior valor de $\mathrm{R}^{2}$ ajustado (quadro 1) e estão entre os seis mais recentes são os que apresentam o EPE e a equação, observaram amostras maiores e, principalmente, realizaram validação cruzada. Algumas considerações devem ser tecidas quanto às vantagens e desvantagens desses estudos: uma tendência nos dois estudos de predição mais recentes ${ }^{11,44}$ foi a utilização apenas de variáveis auto-relatadas no modelo de predição, a fim de diminuir ainda mais o tempo de aplicação. Essa tendência faz com que equações que se valem de variáveis preditivas aferidas, tais como frequiência cardíaca de repouso ${ }^{41}$, apresentem uma grande desvantagem. Isso porque, apesar de as medidas parecerem simples, por vezes demandam 
tempo. No caso da freqüência cardíaca, por exemplo, para ser aferida de maneira precisa é necessário um período de cinco a 10 minutos, mais tempo do que o exigido por alguns testes submáximos. Variáveis que necessitam de aplicadores bem treinados para ser adequadamente coletadas, como as dobras cutâneas, também podem ser desvantajosas na aplicação das equações para estudos com grandes amostras. Em alguns estudos ${ }^{13,14,41}$, o método de dobras cutâneas foi utilizado para predizer o percentual de gordura corporal através de outra equação, o que já pode incluir erros advindos das limitações inerentes a esse tipo de predição.

Um ponto interessante deve ser comentado no caso do estudo de George et al. ${ }^{44}$, já que os próprios autores afirmam que o modelo seria específico para pessoas bem condicionadas. Pode-se pensar, aceitando essa afirmação, que sua aplicabilidade é dirigida a indivíduos que representam uma parte muito pequena da população. Por outro lado, são as pessoas com baixo nível de aptidão cardiorrespiratória que apresentam maior risco de desenvolvimento de doença cardiovascular e metabólica. Considerando esses aspectos, esse modelo poderia ser visto como pouco vantajoso para a pesquisa epidemiológica. Outro importante fator a ser considerado em qualquer estudo que visa à predição da aptidão cardiorrespiratória sem a realização de testes de exercício consiste na influência do componente genético no valor da aptidão. Alguns estudos demonstram que esse componente pode influenciar cerca de 30 a $40 \%$ da magnitude dos resultados ${ }^{50}$. Apesar disso, variáveis relacionadas ao esforço percebido em atividades cotidianas, tais como andar e correr, utilizadas por George et al. ${ }^{44}$, podem surgir como uma alternativa para a avaliação da aptidão cardiorrespiratória sem referência apenas ao histórico da atividade física, uma vez que um indivíduo pode possuir boa aptidão cardiorrespiratória, sem necessariamente praticar exercícios regularmente.

A evolução metodológica dos estudos e a grande precisão dos modelos estabelecidos, em sua maioria para indivíduos saudáveis, sugere que esse tipo de predição consiste em uma boa alternativa para categorizar o nível de aptidão cardiorrespiratória. Contudo, esses modelos ainda continuam a ser pouco utilizados na pesquisa epidemiológica. As equações propostas por Jackson et al. ${ }^{13}$ são as únicas até o momento que foram utilizadas por outros estudos ${ }^{51,52}$. No entanto, a verificação da aplicabilidade dos modelos ainda é escassa. Algumas razões para isso podem ser apontadas. Em primeiro lugar, os modelos têm sido desenvolvidos, em sua maioria, com amostras de indivíduos de nível socioeconômico e cultural médio ou elevado. Como esse perfil não corresponde às características sociais de grande parte da população mundial, as equações vêem restringido seu potencial de generalização, não devendo ser aplicadas indiscriminadamente.

Outro aspecto reside no fato de a variável que descreve a atividade física auto-referida limitar-se, na maioria dos estudos, às atividades de lazer ${ }^{11,13-15}$. Apenas o estudo de Whaley et al..$^{41}$ incluiu em seu modelo, além das atividades de lazer, tarefas de natureza ocupacional. No estudo de Ainsworth et al. ${ }^{10}$, a atividade ocupacional também foi verificada. Entretanto, como foi estudada uma população com atividades ocupacionais sedentárias ou extremamente leves, essa variável não contribuiu para o modelo final de predição. Aliás, populações com baixo nível de atividade ocupacional são encontradas em diversos estudos ${ }^{11,13-15,34}$. Mesmo a população do estudo de Blair et al..$^{12}$, contando com 15.627 homens e 3.943 mulheres, não poderia ser considerada como representativa da população norte-americana, uma vez que realizavam atividades ocupacionais de baixa intensidade, possuíam elevado nível educacional e de médio a elevado nível socioeconômico. Essa limitação, aliás, foi realçada pelos próprios autores ${ }^{12}$. Enfim, o estudo de Wu e Wang ${ }^{46}$, apesar de propor a aplicação do modelo a indivíduos ativos no trabalho, não utiliza nenhuma variável relacionada à atividade física, o que talvez se torne necessário em um grupo maior e mais heterogêneo. Quanto às equações existentes para a predição da aptidão em coronariopatas, pode-se dizer que se propõem a fornecer uma informação adicional sobre a autonomia dos indivíduos. Em regra, não ousam investir-se do poder para predizer a aptidão cardiorrespiratória.

Os estudos sem exercício podem ser comparados a alguns testes submáximos utilizados na pesquisa epidemiológica. Se alguns testes submáximos demonstram um valor de $\mathrm{R}^{2}$ maior do que os sem exercício $(0,81 ; 0,85)$, os valores do EPE são comparáveis $\left( \pm 4 \mathrm{ml} \cdot \mathrm{kg}^{-1} \cdot \mathrm{min}^{-1}\right)^{53}$, ou não são relatados ${ }^{54}$, o que limita a sua utilização. Além disso, em alguns testes submáximos ${ }^{54}$ a validação foi realizada em amostra de faixa etária muito estreita, uma desvantagem que afeta ainda a abrangência da utilização dessas equações. Uma outra questão interessante diz respeito à comparação das equações com questionários de histórico de atividade física, constantemente utilizados na pesquisa epidemiológica. É certo que a natureza e intensidade das atividades cotidianas podem influenciar a aptidão cardiorrespiratória do indivíduo ${ }^{56,57}$. Portanto, ela não deveria ser ignorada como variável dependente no desenvolvimento de modelos de predição. Por outro lado, é geralmente pequena a correlação entre atividade física e aptidão cardiorrespiratória quando baseada em informação de questionários. Quando elevada, a correlação depende quase exclusivamente de questões referentes a atividades físicas intensas. Apesar de a prática de atividade física ser considerada o principal 
determinante da aptidão cardiorrespiratória, questionários sobre atividade física relatada em entrevista ou auto-referida, com duração média de preenchimento entre 15 e 45 minutos, não parecem adequados para avaliar a aptidão cardiorrespiratória ${ }^{39}$. No entanto, esse pode ser um aspecto interessante a ser estudado, pois as equações de predição surgem muitas vezes a partir de questionários simples, cuja força de predição revela-se aumentada ao serem adicionadas variáveis de fácil aferição em modelos mais complexos. As estratégias utilizadas por Mathews et al. ${ }^{11}$ valendose apenas de variáveis auto-relatadas, mesmo no caso de peso e altura, por não necessitarem de muito tempo para a aferição e nem de avaliadores treinados, parecem ser extremamente indicadas para a aplicação para estudos epidemiológicos, e estudos futuros deveriam basear-se nesses princípios.

\section{CONSIDERAÇÕES FINAIS}

Os modelos de predição sem exercício parecem ser um tema que desperta interesse de pesquisadores de todo o mundo. Em princípio, os modelos sem exercícios podem constituir alternativa viável para a avaliação da aptidão cardiorrespiratória em estudos epidemiológicos. Apesar de existirem poucas equações cuja validação permite grau aceitável de generalização, isso reforça a idéia de esta ser uma área extremamente carente e que deve ser explorada através de novos estudos sobre o tema.

Algumas questões prospectivas mereceriam comentário. Em primeiro lugar, para maior aplicabilidade em estudos epidemiológicos, novos estudos não deverão limitar-se apenas à validação e análise transversal dos modelos, passando a voltar-se para a sensibilidade longitudinal da predição do $\dot{V}_{2}$ máximo. Isto é, até o momento não se sabe se alterações nas variáveis preditivas ao longo do tempo (mudanças decorrentes do treinamento) poderão ser detectadas pelos modelos sem exercício. Além disso, é necessário reconhecer que existem poucos estudos especificamente voltados para o desenvolvimento de modelos aplicados a grupos especiais, como (principalmente) idosos, crianças, adolescentes, mulheres ou coronariopatas. Quando existem, têm baixo potencial de generalização, em virtude das amostras reduzidas utilizadas em seu desenvolvimento. Outro aspecto importante é o papel das particularidades socioeconômicas e culturais: a elaboração de modelos de regressão para predizer a aptidão cardiorrespiratória deveria levar em consideração as características da população estudada, principalmente na seleção das variáveis preditivas. É evidente a necessidade de incluir nos estudos a serem validados indivíduos de médio e baixo nível socioeconômico, assim como informações sobre a atividade física exigida pelo trabalho. Essas características tendem a ser específicas para a região ou população observada. Nesse sentido, é latente a necessidade de estudos que se proponham a desenvolver modelos adequados ao contexto das diversas regiões e estratos sociais no Brasil, ou ao menos a verificar a validade de modelos existentes à realidade brasileira.

\section{AGRADECIMENTOS}

Agradecemos aos Doutores Antonio Claudio Lucas da Nóbrega e Antonio Ponce de Leon pelas importantes contribuições e ao graduando Marcelo Moreira Antunes pela tradução do idioma chinês. Estudo parcialmente financiado pela Fundação de Apoio à Pesquisa do Estado do Rio de Janeiro (Processo E-26/151.802/ 1999) e pelo Conselho Nacional de Desenvolvimento Científico e Tecnológico (modalidade Produtividade em Pesquisa, processo 300754/2000-0).

Todos os autores declararam não haver qualquer potencial conflito de interesses referente a este artigo.

\section{REFERÊNCIAS}

1. Caspersen CJ, Powell KE, Christensen GM. Physical activity, exercise, and physical fitness: definitions and distinctions for health-related research. Public Health Rep 1985;100:126-31.

2. American College of Sports Medicine. ACSM's guidelines for exercise testing and prescription. $6^{\text {th }}$ ed. Baltimore: Williams and Wilkins, 2000.

3. Bouchard C, Shephard RJ, Stephens T. Physical activity, fitness, and health: international proceedings and consensus statement. Champaign, IL: Human Kinetics, 1994.

4. Pate RR, Pratt M, Blair SN, Haskell WL, Macera CA, Bouchard C, et al. Physical activity and public health. A recommendation from the Centers for Disease Control and Prevention and the American College of Sports Medicine. JAMA 1995;273:402-7.

5. U.S. Department of Health and Human Services. Physical activity and health: a report of the surgeon general. Atlanta, GA: U.S. Department of Health and Human Services, Centers for Disease Control and Prevention, National Center for Chronic Disease Prevention and Health Promotion, 1996.

6. Lee CD, Blair SN, Jackson AS. Cardiorespiratory fitness, body composition, and all-cause and cardiovascular disease mortality in men. Am J Clin Nutr 1999;69:373-80

7. Wei M, Kampert JB, Barlow CE, Nichaman MZ, Gibbons LW, Paffenbarger RS, et al. Relationship between low cardiorespiratory fitness and mortality in normal-weight, overweight, and obese men. JAMA 1999;282:154753.

8. Delvaux K, Philippaerts R, Lysens R, Vanhees L, Thomis M, Claessens AL, et al. Evaluation of the influence of cardiorespiratory fitness on diverse health risk factors, independent of waist circumference, in 40-year-old Flemish males. Obes Res 2000;8:553-8.

9. Blair SN, Cheng Y, Holder JC. Is physical activity or physical fitness more important in defining health benefits? Med Sci Sports Exerc 2001;33:S37999.

10. Ainsworth BE, Richardson MT, Jacobs DR, Leon AS. Prediction of cardiorespiratory fitness using physical activity questionnaire data. Med Exerc Nutr Health 1992;1:75-82.

11. Mathews CE, Heil DP, Freedson PS, Pastides H. Classification of cardiorespiratory fitness without exercise testing. Med Sci Sports Exerc 1999;31: 486-93. 
12. Blair SN, Kannel WB, Kohl HW, Goodyear N, Wilson PWF. Surrogate measures of physical activity and physical fitness. Am J Epidemiol 1989; 129:1145-56.

13. Jackson AS, Blair SN, Mahar MT, Wier LT, Ross RM, Stuteville JE. Prediction of functional aerobic capacity exercise testing. Med Sci Sports Exerc 1990;22:863-70.

14. Heil DP, Freedson PS, Ahlquist LE, Price J, Rippe J. Nonexercise regression models to estimate peak oxygen consumption. Med Sci Sports Exerc 1995;27:599-606.

15. Kohl HW, Blair SN, Paffenbarger RS Jr, Macera CA, Kronenfeld JJ. A mail survey of physical activity habits as related to measured physical fitness. Am J Epidemiol 1988;127:1228-39.

16. Cotes JE, Davies CTM, Healy MJR. Factors relating to maximum oxygen intake in young adult male subjects. J Physiol 1967;189: 79-80.

17. Cotes JE. Relationships of oxygen consumption, ventilation and cardiac frequency to body weight during standardized submaximal exercise in normal subjects. Ergonomics 1969;12:415-27.

18. Shephard RJ, Weese CH, Merriman JE. Prediction of maximal oxygen intake from anthropometric data. Int Z Angew Physiol 1971;29:119-30.

19. Bruce RA, Kusumi F, Hosmer D. Maximal oxygen and nomographic assessment of functional aerobic impairment in cardiovascular disease. Am Heart J 1973;85:546-62.

20. Mayhew JL, Gifford PB. Prediction of maximal oxygen uptake in pre-adolescent boys from anthropometric parameters. Res Quart 1975;46: 302-11.

21. Bonen A, Heyward VH, Cureton KJ, Boileau RA, Massey BH. Prediction of maximal oxygen uptake in boys, ages 7-15 years. Med Sci Sports 1979;11:24-9.

22. Taylor HL, Jacobs DR, Schucker B, Knudsen J, Leon AS, Debacker G. A questionnaire for the assessment of leisure time physical activities. Journal of Chronic Diseases 1978;31:741-55.

23. Verma SS, Bharadwaj H, Malhotra MS. Prediction of maximal aerobic power in healthy Indian males from anthropometric measurements. Z Morphol Anthropol 1980;71:101-6.

24. Verma SS, Gupta RK, Kishore N, Gupta JS. A simple relationship between aerobic power and body weight in Indian adolescent boys. Indian J Med Sci 1986;40:93-6.

25. Verma SS, Sharma YK, Kishore N. Prediction of maximal aerobic power in healthy Indian males 21-58 years of age. Z Morphol Anthropol 1998;82:10310.

26. Leon AS, Jacobs DR, DeBacker G, Taylor HL. Relationship of physical characteristics and life habits to treadmill capacity. Am J Epidemiol 1981; 113:653-60.

27. Siconolfi SF, Lasater TM, Snow RCK, Carleton RA. Self-reported physical activity compared with maximal oxygen uptake. Am J Epidemiol 1985;122: 101-5.

28. Milesis CA. Prediction of treadmill performance from clinical characteristics in healthy persons. J Cardiopulm Rehabil 1987;7:365-73.

29. Lee TH, Shammash JB, Ribeiro JP, Hartley LH, Sherwood J, Goldman L. Estimation of maximum oxygen uptake from clinical data: performance of the specific activity scale. Am Heart J 1988;115:203-4.

30. Goldman L, Hashimoto B, Cook F, Loscalzo A. Comparative reproducibility and validity of systems for assessing cardiovascular functional class: advantages of a new specific activity scale. Circulation 1981;64:1227-34.

31. Hlatky MA, Boineau RE, Higginbotham MB, Lee KL, Mark DB, Califf $\mathrm{RM}$, et al. A brief self-administered questionnaire to determine functional capacity (the duke activity status index). Am J Cardiol 1989;64:651-4.

32. Astrand PO, Ryhming I. A nomogram for calculation of aerobic capacity (physical fitness) from pulse rate during submaximal work. J Appl Physiol 1954;7:218-21.

33. Kolkhorst FW, Dolgener FA. Nonexercise model fails to predict aerobic capacity in college students with high aerobic $\dot{\mathrm{VO}}_{2 \text { peak }}$. Res Q Exerc Sport 1994;65:78-83.
34. Williford HN, Scharff-Olson M, Wang N, Blessing DL, Smith FH, Duey WJ. Cross-validation of non-exercise predictions of $\dot{\mathrm{VO}}_{2 \text { peak }}$ in women. Med Sci Sports Exerc 1996;28:926-30.

35. Paffenbarger RS Jr, Hyde RT, Wing AL, Hsieh CC. Physical activity, allcause mortality, and longevity of college alumni. N Engl J Med 1986;314: 605-13.

36. Haskell WL, Taylor HL, Wood PD, Schrott H, Heiss G. Strenuous physical activity, treadmill exercise test performance and plasma high-density lipoprotein cholesterol. The Lipid Research Clinics Program Prevalence Study. Circulation 1980;62:IV53-61.

37. Godin G, Shephard RJ. A simple method to assess exercise behavior in the community. Can J Appl Sport Sci 1985;10:141-6.

38. Sallis JF, Haskell WL, Wood PD, Fortmann SP, Rogers T, Blair SN, et al. Physical activity assessment methodology in the Five-City Project. Am J Epidemiol 1985;121:91-106.

39. Jacobs DR Jr, Ainsworth BE, Hartman TJ, Leon, AS. A simultaneous evaluation of 10 commonly used physical activity questionnaires. Med Sci Sports Exerc 1993;25:81-91.

40. Myers J, Do D, Herber W, Ribisl P, Froelicher VF. A nomogram to predict exercise capacity from a specific questionnaire and clinical data. Am J Cardiol 1994;73:591-96.

41. Whaley MH, Kaminsky LA, Dwyer GB, Getchell LH. Failure of predicted $\dot{\mathrm{V}} \mathrm{O}_{2 \text { max }}$ to discriminate physical fitness in epidemiological studies. Med Sci Sports Exerc 1995;27:85-91.

42. Rankin SL, Briffa TG, Morton AR, Hung J. A specific activity questionnaire to measure the functional capacity of cardiac patients. Am J Cardiol 1996;77:1220-3.

43. Cardinal BJ. Predicting cardiorespiratory fitness without exercise testing in epidemiologic studies: a concurrent validity study. J Epidemiol 1996;6:315.

44. George JD, Stone WJ, Burkett, LN. Non-exercise $\dot{\mathrm{VO}}_{2 \max }$ estimation for physically active college students. Med Sci Sports Exerc 1997;22:415-23.

45. Holiday D, Ballard J, McKeown B. PRESS-related statistics: regression tools for cross-validation and case diagnostics. Med Sci Sports Exerc 1995;27:61220.

46. Wu HC, Wang MJJ. Establishing a prediction model of maximal oxygen uptake for young adults. Journal of the Chinese Institute of Industrial Engineers 2002;19:1-7.

47. Greenhalgh T. How to read a paper: statistics for the non-statistician. II: "significant" relations and their pitfalls. BMJ 1997;315:422-5.

48. Altman, D. Practical statistics for medical research. London: Chapman and Hall, 1995.

49. Morrow JR, Jackson AW, Disch JG, Mood DP. Measurement and evaluation in human performance. Champaign, IL: Human Kinetics, 1995.

50. Perusse L, Tremblay A, Leblanc C, Bouchard C. Genetic and environmental influences on level of habitual physical activity and exercise participation. Am J Epidemiol 1989;129:1012-22.

51. Rossy LA, Thayer JF. Fitness and gender-related differences in heart period variability. Psychosom Med 1998;60:773-81.

52. Kelley GA, Lowing L, Kelley K. Gender differences in the aerobic fitness levels of young African-American adults. J Natl Med Assoc 1999;91:384-8.

53. Jette M, Campbell J, Mongeon J, Routhier R. The Canadian home fitness test as a predictor for aerobic capacity. CMAJ 1976;114:680-2.

54. Siconolfi SF, Garber CE, Lasater TM, Carleton RA. A simple, valid step test for estimating maximal oxygen uptake in epidemiologic studies. Am J Epidemiol 1985;121:382-90.

55. Salmon J, Owen N, Bauman A, Schmitz MK, Booth M. Leisure-time, occupational, and household physical activity among professional, skilled, and less-skilled workers and homemakers. Prev Med 2000;30: 191-9.

56. Smolander J, Blair SN, Kohl HW 3rd. Work ability, physical activity, and cardiorespiratory fitness: 2-year results from Project Active. J Occup Environ Med 2000;42:906-10. 